\title{
Acoustic oscillations in stars near the tip of the red giant branch
}

\author{
W. A. Dziembowski ${ }^{1,2}$ and I. Soszyński ${ }^{1}$
}

\author{
1 Warsaw University Observatory, Aleje Ujazdowskie 4, 00-478 Warsaw, Poland \\ e-mail: [wd; soszynsk]@astrouw.edu.pl \\ 2 Copernicus Astronomical Center, ul. Bartycka 4, 00-787 Warsaw, Poland
}

Received 13 June 2010 / Accepted 8 September 2010

\section{ABSTRACT}

\begin{abstract}
Context. Small amplitude oscillations are observed in red giant branch (RGB) stars. Data on such oscillations are a source of information about the objects, notably about properties of convection in their envelopes and about the systems these objects inhabit. The OGLE-III catalog contains data for about 80 thousand small amplitude variable red giants (OSARGs) in the Large Magellanic Cloud. Aims. We want to explain variability oin OSARGs as the solar-like oscillation and to associate the peaks in power spectra with frequencies of acoustic modes.

Methods. We use data on reddening-free magnitudes of the objects and interpret them in terms of stellar physical parameters using tabulated isochrones calculated for ages and composition parameters corresponding to the upper RGB of the LMC. Massive data on the peak frequencies and amplitudes are compared with expectations for stochastically excited oscillations. The frequencies are also compared with those calculated for radial modes in envelope models with parameters taken from the isochrones.

Results. In stars close to the tip of the RGB, the peaks in power spectra are found in the $0.1-1.0 \mu \mathrm{Hz}$ range, which is consistent with extrapolation of the frequency-luminosity relation for the solar-like oscillation. The dominant peaks occur close to the first two radial overtones. The increase in amplitude with luminosity is slower than linear. The exponent $s \approx 0.9$ is similar to what is found from recent analysis of CoRoT data on less luminous red giants. Frequency separations between dominant peaks are found to be smaller by about $20 \%$ than calculated separations between these modes. After examining various possibilities, we left this discrepancy unexplained.

Conclusions. The small amplitude variability of stars at the RGB tip is likely to be caused by a stochastic excitation of acoustic oscillations but interpreting of individual peaks in power spectra presents a problem.
\end{abstract}

Key words. asteroseismology - stars: evolution - stars: late-type - stars: oscillations - Magellanic Clouds

\section{Introduction}

It is now generally accepted that acoustic oscillations in the Sun are stochastically excited by turbulent convection. Oscillations driven in this way, called solar-like oscillations (SLOs), must be excited in all stars having an outer convective zone but, owing to small amplitudes, they are not easy to observe and study. The detection of SLOs in stars along the red giant branch (RGB) was among the most interesting results of asteroseismology with the space telescopes CoRoT (De Ridder et al. 2009) and KEPLER (Stello et al. 2010). Oscillations of this type probe stellar convection, so it is important to detect them in objects over a possibly wide range of surface parameters. Data on mode frequencies in individual red giants could give us important constraints on evolutionary models. This requires, however, a credible mode identification.

The signature of SLOs is an excess of power around a given frequency $v_{\max }$, which decreases with growing stellar luminosity. The $L^{-1}$ decrease was expected (Kjeldsen \& Bedding 1995) and partially confirmed by observations. Data from CoRoT (Mosser et al. 2010) and KEPLER (Stello et al. 2010) reach down to $v_{\max } \approx 2 \mu \mathrm{Hz}$, which roughly corresponds to $L=300 L_{\odot}$. This number is about one order of magnitude above the red clump composed of helium-burning stars and one order of magnitude below the RGB tip (TRGB), which stars reach at the moment of helium ignition.

On a large scale, small amplitude oscillations in luminous RGB and asymptotic giant branch (AGB) stars were detected as a byproduct of microlensing surveys MACHO and OGLE. Using data from the first survey, Wood et al. (1999) and Wood (2000) show that the LMC red giants form five ridges (sequences) in the period-luminosity plane. The ones labeled A and B were associated with the small amplitude pulsators. Later, the MACHO data were analyzed by Fraser et al. (2005, 2008). Data from the OGLE-II survey for both Magellanic Clouds were the subject of independent analyses by Kiss \& Bedding (2003, 2004), Soszyński et al. (2004), and Ita et al. (2004a,b). Additional period-luminosity sequences formed by low amplitude objects were identified by these authors. Interpretations of these sequences in terms of radial pulsation modes in RGB and AGB stars were considered. Evidence for the multiperiodic character of pulsation was found in both sets of data.

Here we use the acronym OSARGs OGLE small amplitude red giants, which was introduced by Wray et al. (2004) in their paper on variability of red giants in the Galactic Bulge. Soszyński et al. (2004), who discovered a large number of OSARGs in the Magellanic Clouds (15 400 in LMC and 3000 in SMC), showed that they fall into seven detached ridges in the period-luminosity plane. Four of the ridges extending beyond the TRGB were attributed to the AGB stars. These objects were named type a OSARGs. The ones lying within the three ridges ending at TRGB were regarded genuine RGB stars and named type b OSARGs.

The OGLE-III Catalog of Variable Stars (Soszyński et al. 2009) contains data for about $8 \times 10^{4}$ OSARGs in the Large 
OSARGs a (AGB)

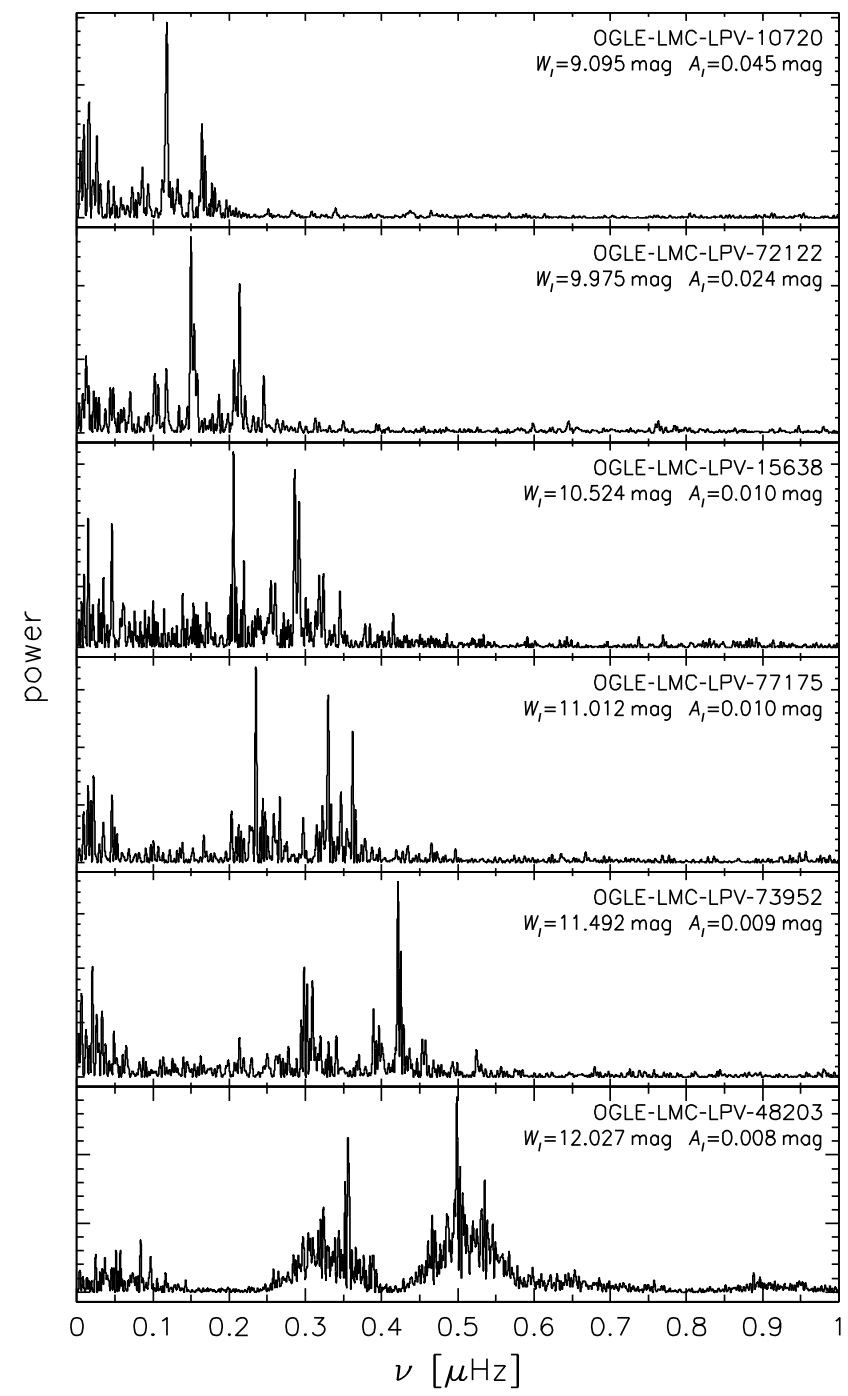

OSARGS b (RGB)

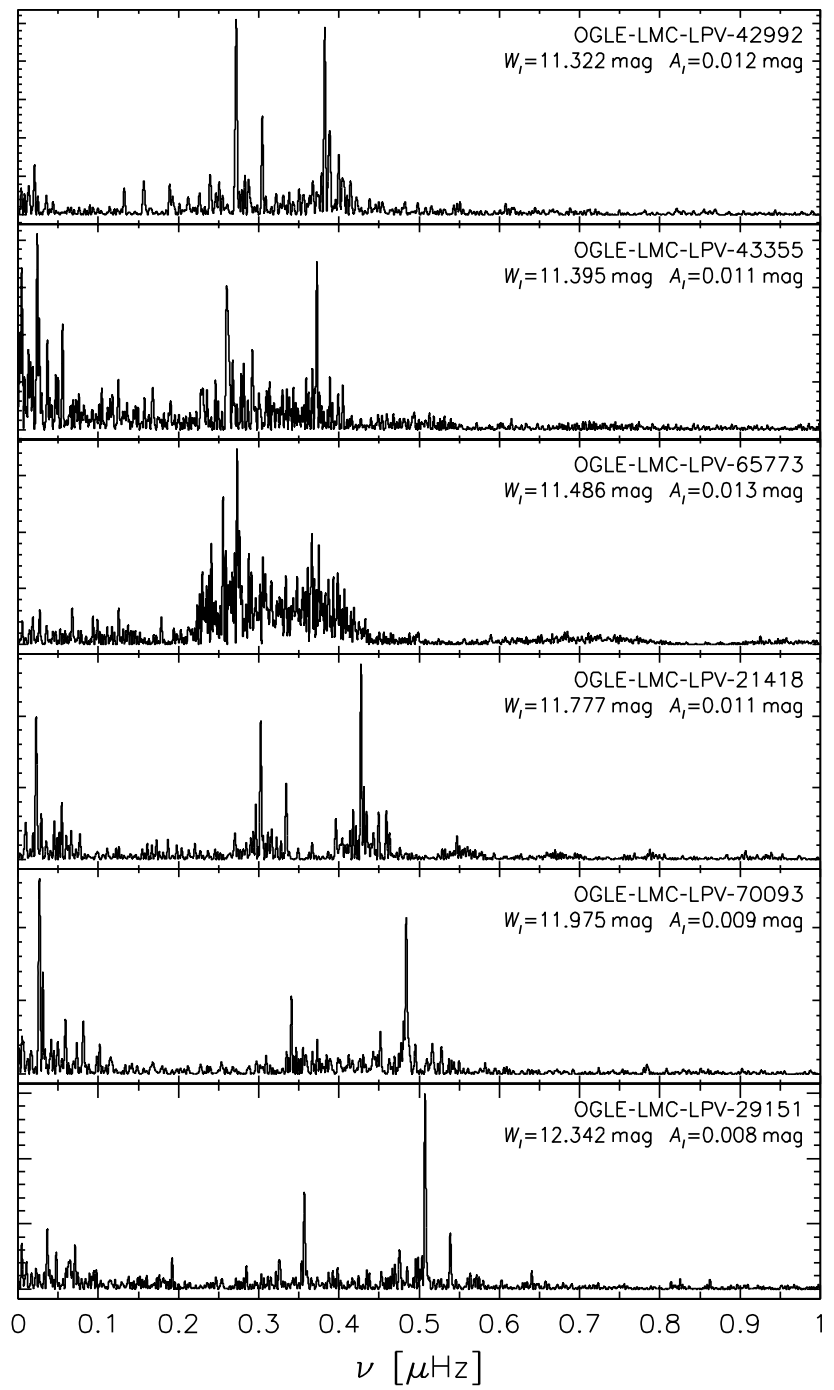

Fig. 1. A sample of power spectra of a and b type OSARGs. For each spectrum we give the OGLE identification number, the Wesenheit index ( $W_{I}$, see Eq. (1)), and the amplitude of the highest peak in the the $I$-band $\left(A_{I}\right)$.

Magellanic Cloud (LMC) and is based on photometric data collected during 8 years (2001-2009) of continuous observations within the framework of the OGLE-III project. For some of the objects, the time base was increased to 13 years thanks to photometry from the OGLE-II project. In this paper, we focus mainly on type $b$, because we only have adequate models for RGB stars. First, in a phenomenological way, we address the question how the OSARGs' variability is driven. Next, we present an attempt to explain the dominant peaks in terms of stellar oscillation modes.

\section{Are the OSARGs solar-like pulsators?}

The origin of OSARGs is not a new question. It has been already addressed by Soszyński et al. (2007), but some arguments in favor of such an interpretation were presented in a relatively short section, which went unnoticed by people working on SLOs.

Stochastic excitation must take place in red giants, but it is not at all clear whether the observed variability of OSARGs is predominantly caused by this mechanism. In fact, Xiong \& Deng (2007) find that some low-order radial modes are unstable in RGB stars. This instability arises mainly from a small excess of the driving effect of turbulent pressure over damping effect of the turbulent viscosity. Both effects originate in the perturbed Raynolds stress, and are difficult to estimate. Thus both the stochastic excitation and the self-excitation of unstable modes must be regarded as viable suggestions. In the wake of uncertainties in the description of convection in red giants, we propose a closer look at observational data to see how the properties of low-amplitude oscillations in luminous red giants follow the trends seen in fainter objects.

We began by comparing patterns in power spectra. Figure 1 shows examples of spectra for OSARGs, based on the OGLE $I$-band photometry. We chose the ones that have prominent peaks contributing to $a_{2}, a_{3}, b_{2}, b_{3}$ sequences (Soszyński et al. 2007), which are the most prominent in the $P L$-plane. The patterns seen in this figure are reminiscent of those shown in Fig. 1 of De Ridder et al. (2009) or Fig. 2 of Stello et al. (2010), except that the frequency range is a factor 100 lower. In all periodograms shown in our Fig. 1, we see the two prominent peaks that were the feature behind our selection rule, but besides this, there is considerable diversity, especially in the amount of power around the dominant peaks and in the presence of the secondary peaks. In all cases there is a power localized in some 
W. A. Dziembowski and I. Soszyński: Acoustic oscillations in stars near the tip of the red giant branch

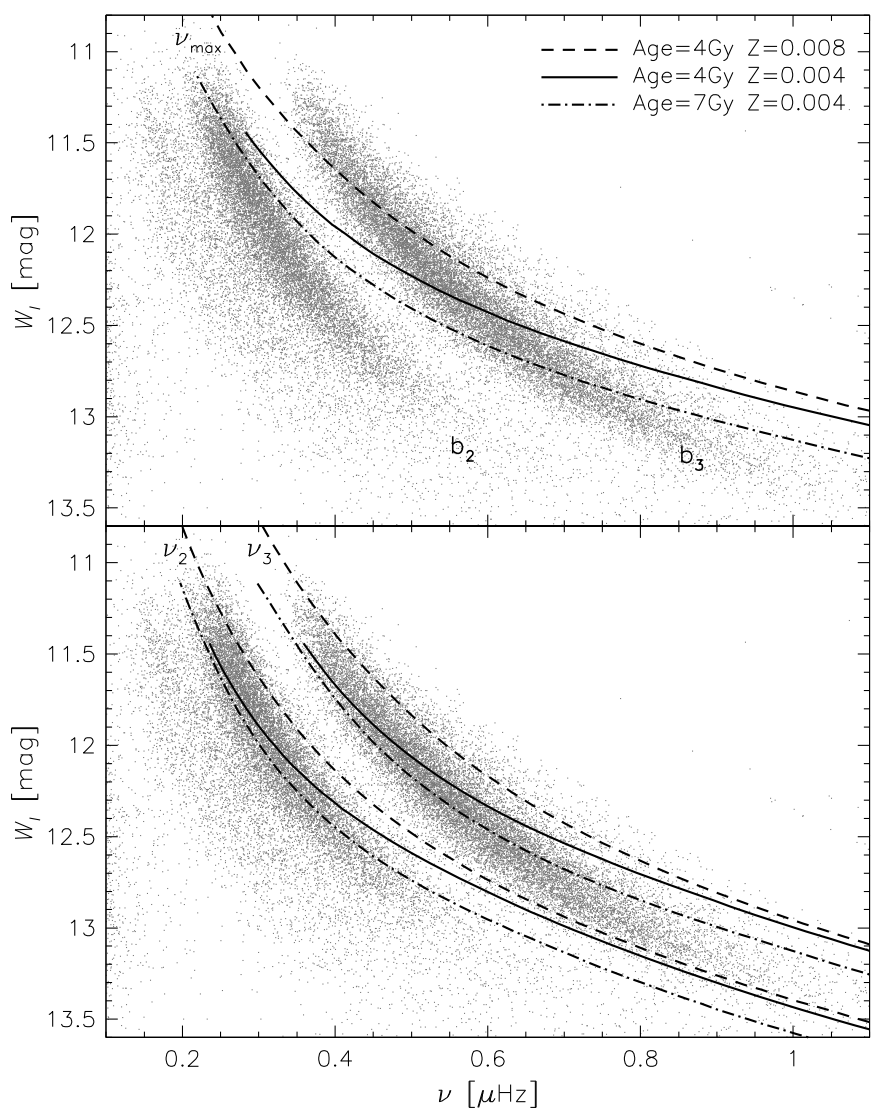

Fig. 2. The $b_{1}$ and $b_{2}$ sequences in the $v-W_{I}$ plane compared with the expected positions of power calculated with Eq. (2) (top) and with frequencies of the first two radial overtones, $v_{2}$ and $v_{3}$ (bottom). The dots show all significant peaks at the $S / N=5$ level. Some objects yield points on both sequences.

intermediate frequency range that moves leftwards with increasing luminosity. Simultaneously, the separation between dominant peaks decreases. These are features seen in all power spectra of SLOs.

The low-frequency power in the OGLE data may be attributed to the granulation noise, like in other cool stars. However, the high-amplitude peaks in this range come from well-known but still unexplained phenomenon of the long secondary period (LSP) observed in a significant fraction $(\sim 30 \%)$ of red giants (see, e.g., Nicholls et al. 2009). The power spectra shown in this figure are very similar to those shown by Tabur et al. (2010) for bright galactic M giants. Some of those objects are evidently located near TRGB.

We turn now to data on all OSARGs that contribute to the sequences $b_{2}$ and $b_{3}$. From the OGLE-III Catalog, we took the respective frequencies and the Wesenheit index, $W_{I}$, for individual objects. This index, which is the reddening-free stellar magnitude, is defined as

$W_{I}=I-1.55(V-I)$,

where $V$ and $I$ denote mean magnitudes in the respective bands. Points in Fig. 2 present data on the most significant periodicities $(S / N \geq 5)$. Up to two points are plotted per star. To compare these data with information on SLOs in less luminous stars, we need stellar parameters, such as luminosity, effective temperature, and mass. To this end, we used isochrones downloaded from the BaSTI Library (Pietrinferni et al. 2006) for ages and metal abundance parameters appropriate to stars at TRGB in
Table 1. Selected isochrones and stellar parameters at $W_{I}=12$.

\begin{tabular}{ccccccl}
\hline \hline Age [Gy] & {$[M / \mathrm{H}]$} & $Z$ & $Y$ & $M / M_{\odot}$ & $L / L_{\odot}$ & $T_{\text {eff }} / T_{\text {eff } \odot}$ \\
\hline 7 & -0.659 & 0.004 & 0.251 & 0.965 & 1795 & 0.657 \\
4 & -0.659 & 0.004 & 0.251 & 1.145 & 1945 & 0.658 \\
4 & -0.953 & 0.008 & 0.256 & 1.222 & 1563 & 0.642 \\
\hline
\end{tabular}

the LMC (Salaris \& Girardi 2005). For the distance modulus to LMC, we adopted $18.5 \mathrm{mag}$, which is close to the mean value from recent determinations by various methods (Schaefer 2008). The characteristics of the selected isochrones and model parameters at the point where $W_{I}=12$ mag are listed in Table 1 . In the range covered by the data, there is about factor 4 increase in luminosity between the highest and the lowest frequencies.

The three lines in the upper panel of Fig. 2 were calculated along the isochrones with the Kjeldsen \& Bedding (1995) expression

$v_{\max }=\frac{L_{\odot}}{L} \frac{M}{M_{\odot}}\left(\frac{T}{T_{\odot}}\right)^{3.5} \times 3050 \mu \mathrm{Hz}$

for the frequency corresponding to maximum of the acoustic power. This expression is consistent with data on solar-like oscillation from main sequence up to lower RGB (Bedding \& Kjelsen 2003; Stello et al. 2007). We may see that it also approximately applies to our data, which extend up to TRGB. This is an argument in favor of the solar-like nature of oscillations in type $b$ OSARGs. but the possibility of self-excitation cannot be ruled out. The argument for of the latter interpretation is that peaks are narrow, suggesting long-lived modes.

In the lower panel we show the frequency-luminosity dependence for the first and second overtones along the three isochrones. The frequencies were calculated with our nonadiabatic pulsation code (Dziembowski 1977) for envelope models with surface parameters from the isochrones ${ }^{1}$. The data suggest that $b_{2}$ and $b_{3}$ sequences may be connected with excitation of these two overtones. The frequency of the specified mode is roughly proportional to the square of mean density, hence $v_{k} \propto L^{-3 / 4}$. while $v_{\max } \propto L$. Therefore, with increasing luminosity, the order of modes preferred by SLOs decreases.

Another characteristic of the SLOs is the dependence of oscillation amplitudes on stellar parameters. Kjeldsen \& Bedding (1995) derived a semi-empirical expression for the bolometric luminosity amplitude of $\delta L \propto(L / M) T_{\mathrm{eff}}^{-2}$. Samadi et al. (2007) considered a more general dependence of amplitude on the luminosity-to-mass ratio, $(L / M)^{s}$. They show that the $s$-exponent ( $s v$ in their notation) is determined by the eddy time-correlation form. The Gaussian form yields $s=1$, while the Lorentzian $s=0.7$. They also show that the latter value fits the groundbased data on radial velocity amplitudes better. The space data on luminosity amplitudes yield discrepant conclusions. Stello et al. (2010) find $s \approx 0.7$ from the Kepler data, while Mosser et al. (2010) found $s=0.89 \pm 0.02$ from their analysis of the CoRoT data.

The OGLE-III Catalog contains data on mode amplitudes in the $I$ band. We compared these data with the prediction from

1 The parameters do not include the surface helium abundance, which in the RGB stars may be up to ten percent higher than the initial one because of the dredge-up of the nuclear processed gas. We checked that such an increase has no visible effect on the plots shown in this paper. 


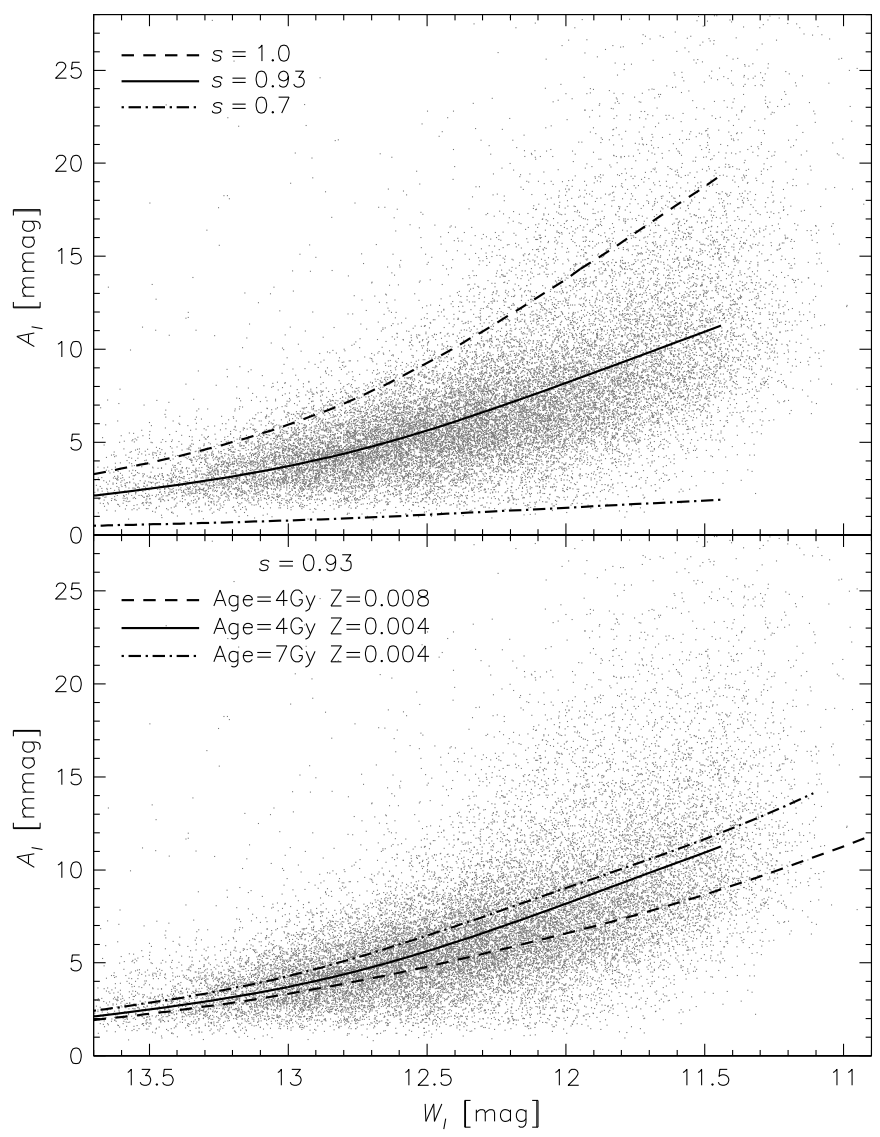

Fig. 3. The highet amplitudes of the peaks on the $b_{2}$ or $b_{3}$ sequences as a function of the reddening-free magnitude $W_{I}$ compared with the expected values for the solar-like oscillations calculated with Eq. (3). In the upper panel, the results of calculations with the three indicated values of $s$ for models along one of the three isochrones (Age $=$ $4 \mathrm{~Gy}, Z=0.004$ ) are shown. In the lower panel, results for the three isochrones at $s=0.93$ are compared.

a generalized form of Eq. (8) of Kjeldsen \& Bedding (1995), which at $\lambda=800 \mathrm{~nm}$ yields

$A_{I}=\left(\frac{L}{L_{\odot}} \frac{M_{\odot}}{M}\right)^{s}\left(\frac{T_{\odot}}{T}\right)^{2} \times 0.0035 \mathrm{mmag}$.

The plots in Fig. 3 show that the OGLE data follow the relation with $s \approx 0.9$. Both, $s=0.7$ and 1 are excluded. Our result is, thus, similar to that of Mosser et al. (2010).

\section{Modes excited in luminous red giants}

Identification of modes responsible for the peaks in power spectra such as those shown in Fig. 1 would give us a basis for a real seismic probing of individual luminous red giants. The plots in the bottom panel of Fig. 2 suggest that the dominant peaks are associated with the first and second overtones of radial pulsation. If it was true, the frequency distance between the peaks would be the large separation, $\Delta v$. In lower luminosity stars, more than one large separation is measured. Their mean value, $\langle\Delta v\rangle$, is regarded as an important seismic parameter. However, the mean distance between sequences $b_{2}$ and $b_{3}$ at specified $W_{I}$ cannot be identified with $\langle\Delta v\rangle$ because it does not refer to the same object. We need the distance between the dominant peaks measured separately in individual power spectra. With this in mind, we selected over 100 objects with the most significant $(S / N \geq 7)$ peaks within the

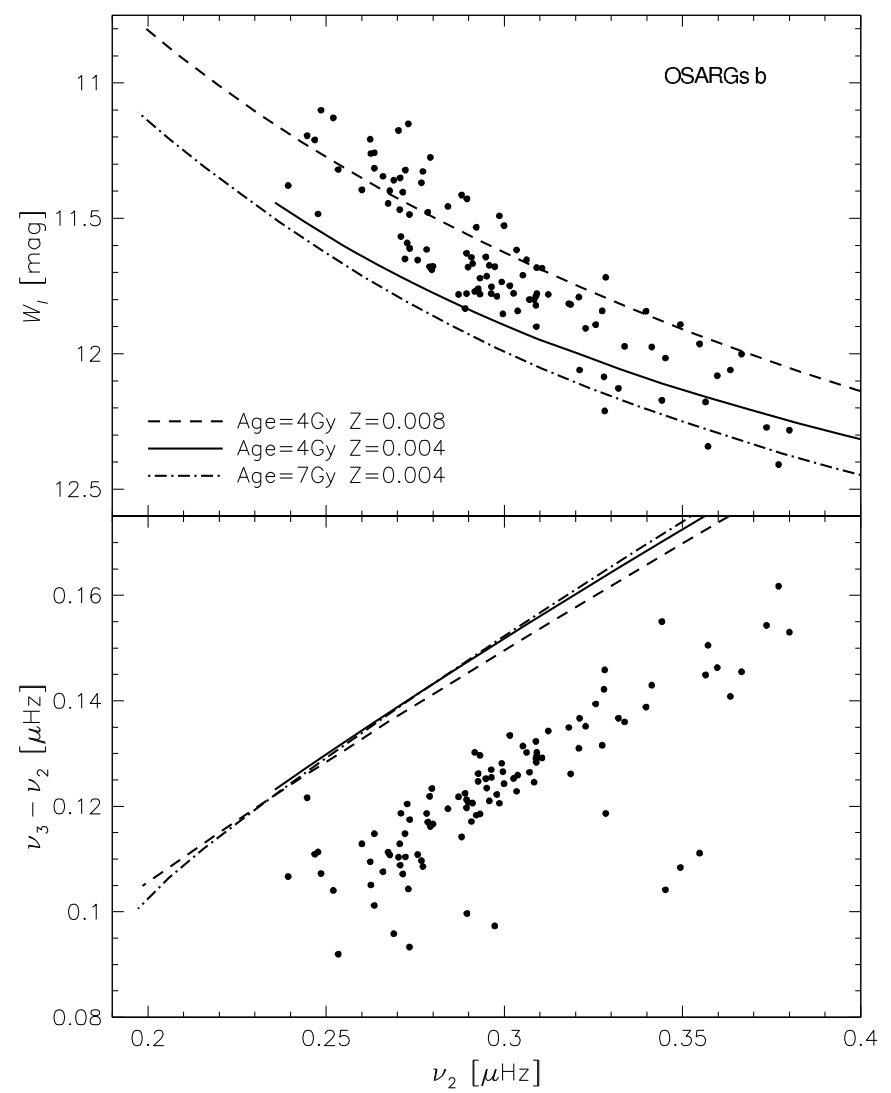

Fig. 4. Frequencies of the most significant peaks within the $b_{2}$ and $b_{3}$ sequences in selected objects (dots) compared with frequencies of the first two overtones in the models along the three isochrones. The top panel shows the reddening-free magnitude $W_{I}$ as a function of the lower frequency. The bottom one shows the frequency difference.

two sequences. Plots in Fig. 4 show that, although the observed $W_{I}\left(v_{2}\right)$ relation is reproduced well by the models, the difference between the frequencies, which is shown in the lower panel, is by some 20 percent less than the calculated difference between the second and the first overtones. This difference is quite a robust quantity depending almost only on $v_{1}$. It is only weakly dependent on the isochrone parameters, as may be seen in the figure, as well as on details of modeling the envelope and its pulsation.

Our first suspicion has been that the discrepancy may be due to inadequacies of our codes, which were written long ago (Paczyński 1969; Dziembowski 1977). The microphysics has been updated, but still the turbulent pressure and the Lagrangian perturbation of the convective flux are ignored in these codes. Fortunately, Xiong \& Deng (2004) have provided a functional expression for radial mode frequencies calculated with their much more advanced treatment of convection for a sequence of red giant envelope models. Repeating calculation with our code for models with the same surface parameters, we found only a small difference, which was much too small to explain the discrepancy shown in Fig. 4. Also acceptable departures from the mixing length parameter adopted in the evolutionary models only have a minor effect on the dependence of the $v_{3}-v_{2}$ difference on $v_{2}$.

The problem with interpreting the $b_{2}$ and $b_{3}$ sequences in terms of the first two overtones could have been noticed earlier with a closer look at the Petersen Diagrams shown by Soszyński et al. (2004). In such diagrams, period ratios of all significant peaks detected in individual objects are plotted against the longer 
W. A. Dziembowski and I. Soszyński: Acoustic oscillations in stars near the tip of the red giant branch

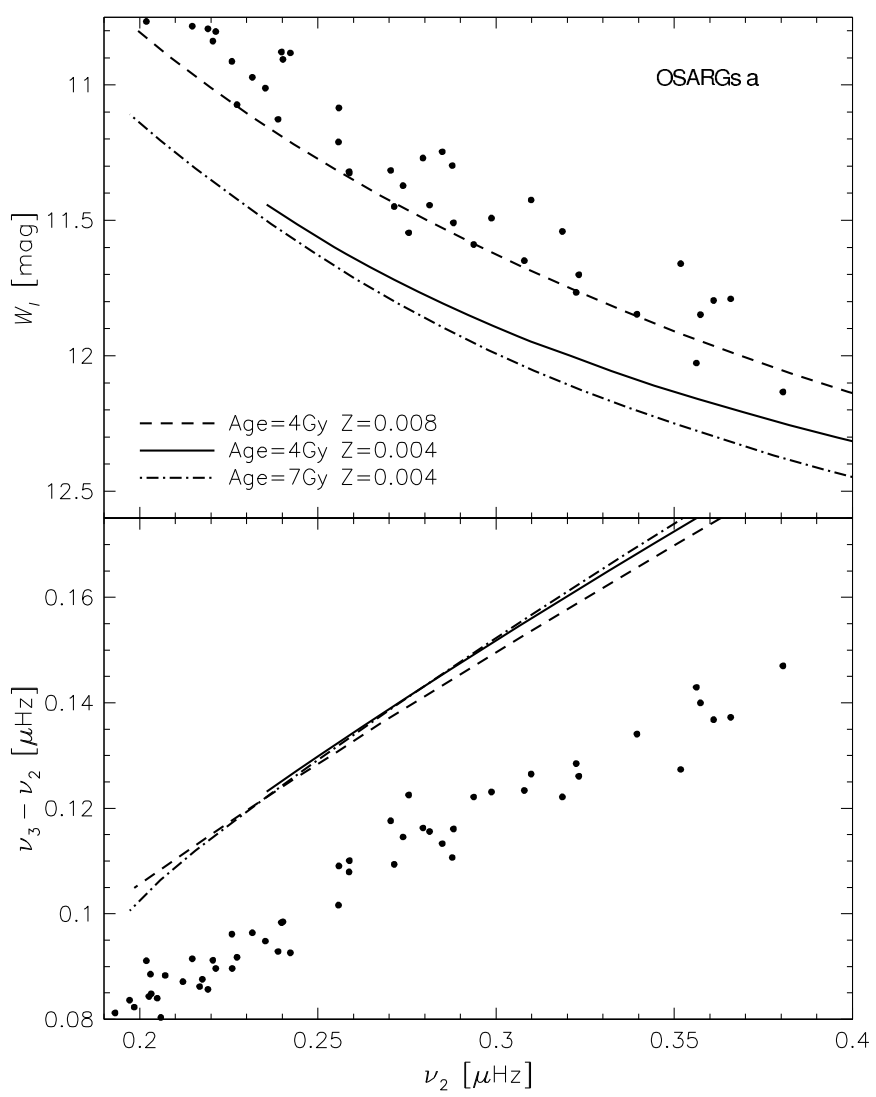

Fig. 5. Same as Fig. 4 but for peaks within the $a_{2}$ and $a_{3}$ sequences.

periods. In Fig. 11 of that paper, we may see a prominent cloud of points corresponding to the $b_{2}$ and $b_{3}$ sequences near $P_{2} / P_{3} \approx$ 0.7. However, most of them are located above this value, whereas in the same period range, the models yield values that are always below 0.7. The mean value for the cloud of 0.73 is not reached until $v_{2} \approx 1 \mu \mathrm{Hz}$.

We focused on type b OSARGs because they are, as we believe, RGB objects, and we lack needed models of AGB stars. Nonetheless, it is still instructive to compare frequencies of type a OSARGs with the values calculated along the isochrones in the same range of the $W_{I}$ magnitudes. The essential difference between AGB and RGB stars concerns the deep interior, which has no effect on radial mode frequencies. The former objects have helium-exhausted cores, while the latter are either in the phase of core helium burning or still ahead of it. In Fig. 5, we compare data on type a OSARGs with results of the the same calculations as were used for type b. Points in the upper panel seem to follow a single isochrone, which should be somewhat younger and/or have higher $Z$ than that for $Z=0.008$. This suggests that at least some of type a OSARGs located below TRGB are in fact the first ascent objects before helium ignition. The data on frequency differences shown in the lower panel reveal the same problem with interpretation in terms of the frequency difference between the first two radial overtones. The discrepancy is only somewhat smaller but there is much less spread in the data. So the problem is not less severe.

We regard the discrepancy revealed in Figs. 4 and 5 as serious. Any other assignment of radial mode orders makes the discrepancy worse. If no modification in stellar models can remove the problem with interpretation in terms of the radial modes, the option is to consider non-radial modes trapped in the envelope. Such modes may avoid large radiative losses in the interior

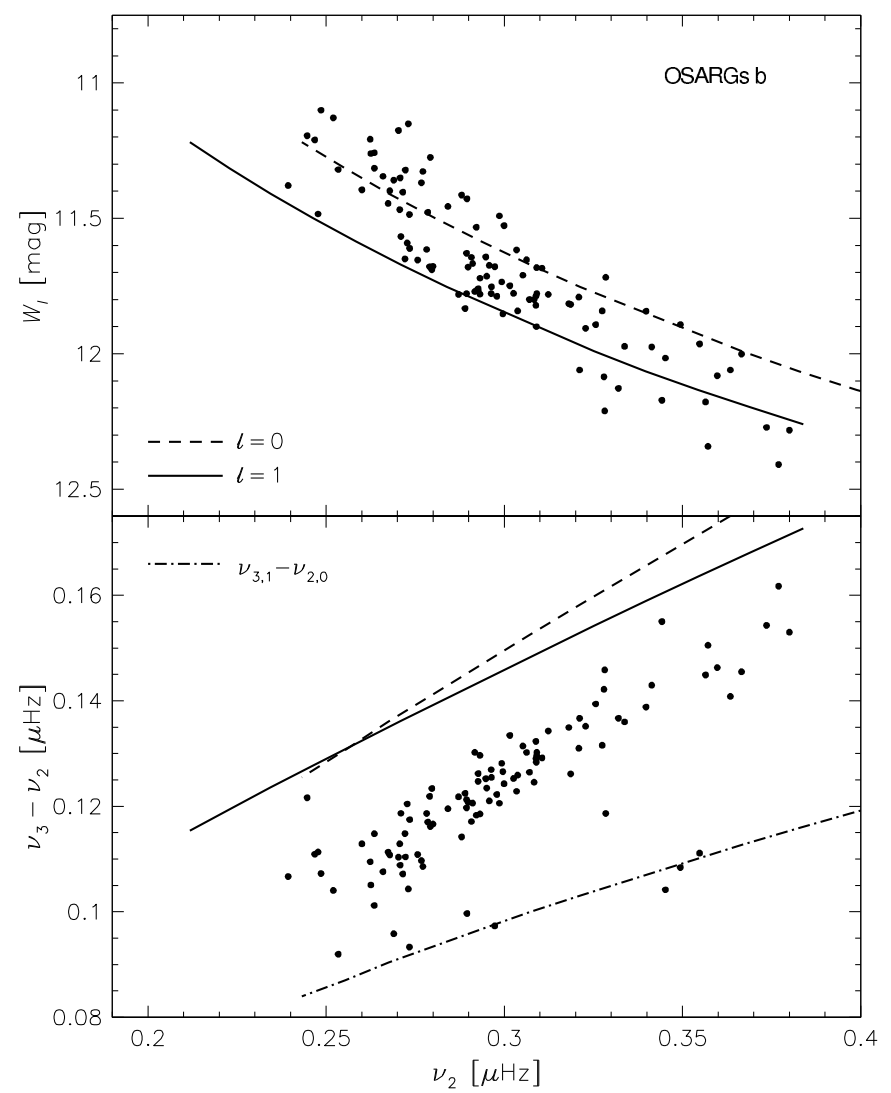

Fig. 6. Same data as in Fig. 4 compared with frequencies of radial $(\ell=$ $0)$ and dipolar $(\ell=1)$ modes in models along the Age $=4 \mathrm{~Gy}, Z=$ 0.008 isochrone. The second subscript at $v$ corresponds to the angular degree, $\ell$.

(Dziembowski et al. 2001; Dupret et al. 2009). Though it is difficult to explain why such modes could be preferentially excited, we did consider this possibility. We limited ourselves to dipolar modes because they exhibit relatively small amplitude reduction caused by cancelation of opposite sign contributions to disk-averaged value. The plots in Fig. 6 show that allowing dipolar mode interpretation of the dominant peaks does not solve the problem ${ }^{2}$. The vast majority of the data points shown in the lower panel falls far from any of the three lines representing calculated frequency differences.

\section{Discussion and conclusions}

Small-amplitude variability is a common phenomenon in all stars along the RGB. Data from the ongoing space missions CoRoT and Kepler filled the gap that existed between lowluminosity objects, whose oscillations were found in radial velocity data, and ones located near the tip detected by means of massive photometry. The small-amplitude variability observed at lower luminosity is always interpreted, as in the Sun, as a manifestation of a stochastic excitation of acoustic modes.

The first question we addressed in this paper was whether oscillations observed in the high-luminosity red giants from the OGLE-III catalog (OSARGs) follow the pattern found at lower luminosity. We showed that for these stars the oscillation power is concentrated in the frequency range of the first two overtones,

2 The dipolar mode frequencies were calculated with a new version of our code for oscillations in unfitted envelope models that does not assume the Cowling approximation. 
which is consistent with extrapolation of the $v_{\max }(L)$ relation established for less luminous objects. For the solar-like oscillations, amplitude increase with luminosity is described by the $A \propto L^{s}$ relation, with $s$ in the [0.7-1.0] range. Samadi et al. (2007) show that the value $s$ depends on properties of turbulence, which drives the oscillations, and that data for low-luminosity objects are best fitted with $s \approx 0.7$. De Ridder et al. (2009) obtained a similar result for more luminous stars. Our value $s \approx 0.9$ for the OGLE-III sample of RGB stars is similar to the one found by Mosser et al. (2010) for fainter objects. These arguments, in addition to the apparent similarity of power spectra shown in Fig. 1, support the idea that the variability of stars near the tip of RGB is caused by a stochastic excitation of acoustic modes.

We encountered a problem in our effort to assign radial mode frequencies to the two dominant peaks in the OSARGs power spectra. The observed frequency dependence on luminosity is not far from what is expected for first two radial overtones, but the frequency difference is by some $20 \%$ less than that calculated for the models. The calculated frequency difference was found to be only weakly dependent on model parameters. We could not find any reasonable modification in models that could lead us in the right direction. We checked that the frequencies calculated with our code are in good agreement with ones calculated for stellar envelope models with the same surface parameters by Xiong \& Deng (2007). We considered the possibility that one or both peaks are stem from the dipolar mode excitation and found that this does not solve the problem either.

Our failure does not mean the dominant peaks in OSARGs' power spectra cannot be associated with low-degree modes of stellar oscillations. The problem may still be caused by inadequacy of our models. We do encourage further efforts along this line because eigenmode frequencies are indeed the most important seismic observables.

The alternative. which may seem less attractive, is that the peaks are not caused by stellar normal mode excitation. Most of narrow peaks in red giants' power spectra from the space missions have not been associated with stellar modes either. However, even if this is so, the data on red giant variability remain of considerable interest for stellar physics. They certainly carry information that deserves to be extracted. In the case of OSARGs, the data demanding interpretation are the seven ridges in the frequency-magnitude plane.

Acknowledgements. Part of this work was supported by the Polish MNiSW grant number N N203 379636.

\section{References}

Bedding, T. R., \& Kjeldsen, H. 2003, PASA, 20, 203

De Ridder, J., Barban, D., Baudin, F., et al. 2009, Nature, 459, 398

Dupret, M. A., Belkacem, K., Samadi, R., et al. 2009, A\&A, 506, 57

Dziembowski, W. 1977, Acta Astron., 27, 95

Dziembowski, W. A., Gough, D. O., Houdek, G., \& Sienkiewicz, R. 2001, MNRAS, 328, 601

Fraser, O. J., Hawley, S. L., Cook, K. H., \& Keller, S. C. 2005, AJ, 129, 768

Fraser, O. J., Hawley, S. L., \& Cook, K. H. 2008, AJ, 136, 1242

Ita, Y., Tanabé, T., Matsunaga, N., et al. 2004a, MNRAS, 347, 720

Ita, Y., Tanabé, T., Matsunaga, N., et al. 2004b, MNRAS, 353, 705

Kiss, L. L., \& Bedding, T. R. 2003, MNRAS, 343, L79

Kiss, L. L., \& Bedding, T. R. 2004, MNRAS, 347, L83

Kjeldsen, H., \& Bedding, T. R. 1995, A\&A, 293, 87

Mosser, B., Belkacem, K., Goupil, M.-J., et al. 2010, A\&A, 517, 22

Nicholls, C. P., Wood, P. R., Cioni, M.-R. L., \& Soszyński, I. 2009, MNRAS, 399,2063

Paczyński, B. 1969, Acta Astron., 19, 1

Pietrinferni, A., Cassisi, S., Salaris, M., \& Castelli, F. 2006, ApJ, 642, 797

Salaris, M., \& Girardi, I. 2005, MNRAS, 357, 669

Samadi, R., Georgobiani, D., Trempedach, R., et al. 2007, A\&A, 463, 297

Schaefer, B. E. 2008, AJ, 135, 112

Soszyński, I., Udalski, A., Kubiak, M., et al. 2004, Acta Astron., 54, 129

Soszyński, I., Dziembowski, W. A., Udalski, A., et al. 2007, Acta Astron., 57, 1

Soszyński, I., Udalski, A., Szymański, M., et al. 2009, Acta Astron., 59, 239

Stello, D., Bruntt, H., Kjeldsen, H., et al. 2007, MNRAS, 377, 584

Stello, D., Basu, S., Bruntt, H., et al. 2010, ApJ, 713, 182

Tabur, V., Bedding, T. R., Kiss, L. L., et al. 2010, MNRAS, 409, 777

Wood, P. R. 2000, Publ. Astron. Soc. Aust., 17, 18

Wood, P. R., Alcock, C., Allsman, R. A., et al. 1999, IAU Symp., 191, 151

Wray, J. J., Eyer, L., \& Paczyński, B. 2004, MNRAS, 349, 1059

Xiong, D. R., \& Deng, I. 2007, MNRAS, 378, 1270 